\title{
13-cis-Retinoic Acid in Combination with Gemcitabine in the Treatment of Locally Advanced and Metastatic Pancreatic Cancer - Report of a Pilot Phase II Study
}

\author{
A. Michael*, M. Hill†, A. Maraveyas $\ddagger$, A. Dalgleish*, F. Lofts* \\ *Oncology Department, St George's Hospital, St James' Wing, Blackshaw Road, London SW17 0QT, UK; †Maidstone General Hospital, \\ Hermitage Lane, Maidstone, Kent ME16 9QQ UK; †Princess Royal Hospital, Salthouse Road, Hull HU8 9HE, UK
}

\begin{abstract}
:
Aims: Adenocarcinoma of the pancreas is a cancer with extremely poor prognosis and limited therapeutic options. Retinoids are derivatives of vitamin A involved in the control of many biological functions, including cell growth and differentiation and the induction of apoptosis. On the basis of pre-clinical evidence and some clinical data, we conducted a phase II study of 13-cis-retinoic acid (13-cis-RA) in combination with gemcitabine in patients with unresectable pancreatic carcinoma.

Materials and methods: Patients with histologically confirmed unresectable pancreatic carcinoma were treated with gemcitabine $1000 \mathrm{mg} / \mathrm{m}^{2}$ on days $8,15,22$ plus $13-$ cis-RA $1 \mathrm{mg} / \mathrm{kg}$ on days $1-14$ for six cycles. The end points included the objective response rate and median survival.

Results: Thirty patients were recruited, 15 men and 15 women; 20 patients were evaluable. The median age was 65 years (range 44-79 years) and the median Karnofsky performance status was $80 \%$ (range $60-100 \%$ ). The median followup was 21 months. One patient achieved a partial remission, seven patients had stable disease and 12 patients developed progressive disease. Toxicity was mainly haematological, with eight cases of grade 3 and four cases of grade 4 neutropenia, thrombocytopenia and anaemia. The median survival was 7.8 months (range 2.6-21.6 months).

Conclusions: The combination of gemcitabine and 13-cis-RA was well tolerated, but we did not see improvement in the response rate. Further studies with other retinoids may be beneficial to patients with unresectable pancreatic cancer. Michael, A. et al. (2007). Clinical Oncology 19, 150-153
\end{abstract}

(c) 2006 The Royal College of Radiologists. Published by Elsevier Ltd. All rights reserved.

Key words: 13-cis-Retinoic acid, gemcitabine, pancreatic cancer

\section{Introduction}

Adenocarcinoma of the pancreas is one of the most common causes of cancer death in the Western world. In the UK, only $2 \%$ of men and $3 \%$ of women diagnosed with pancreatic cancer survive for 5 years [1]. The most accepted and least toxic chemotherapy agent currently used for pancreatic cancer is gemcitabine. It offers a small survival advantage and is effective in improving some disease-related symptoms [2]. A search for novel therapeutic agents that would increase the activity or act in synergy with chemotherapy drugs has resulted in a number of potential approaches. One of them concentrated on the use of retinoids in cancer. Retinoids are derivatives of vitamin A and are involved in the control of many biological functions, including cell growth and differentiation and the induction of apoptosis [3-6]. Retinoids exert their effect by binding to the nuclear receptors, functioning as ligand-dependent transcription factors [7]. There are two families of receptors binding retinoic acids (RA): the RA receptors (RARs) and the retinoid $X$ receptors (RXRs). The natural ligand for RARs is all-trans retinoic acid (ATRA); RA, such as 9-cis-RA, binds to both ligands, RARs and RXRs [8]. There is ample pre-clinical evidence that retinoids are active against pancreatic cancer cell lines [9-13]. The synergistic effect of the combination of 9-cis-RA with gemcitabine on pancreatic cancer cell lines has been explored by Pettersson et al. [14]. The pretreatment of pancreatic adenocarcinoma cells with ATRA or 9-cis-RA before the addition of gemcitabine resulted in a significant increase in the number of apoptotic cells as compared with chemotherapy alone. 13-cis-RA has been tested in combination with interferon- $\alpha$ in a clinical study and the results were encouraging [15]. On the basis of this evidence, we designed a phase II pilot study to investigate a combination therapy of 13-cis-RA and gemcitabine in patients with unresectable pancreatic carcinoma. 


\section{Patients and Methods}

Patients with histologically confirmed unresectable pancreatic adenocarcinoma were recruited from three oncology centres. All patients needed to have a life expectancy of at least 12 weeks and adequate haematological and liver function, as defined by haemoglobin $>10 \mathrm{~g} / \mathrm{dl}$, absolute neutrophil count $>1.5 \times 10^{9} / \mathrm{l}$, platelet count $>100000 /$ $\mathrm{mm}^{3}$, total bilirubin $<50 \mathrm{mmol} / \mathrm{l}$ and Alanine aminotransferase (ALT), Aspartate aminotransferase (AST) and alkaline phosphatase $<5 \times$ upper limit of normal. Patients had to have a Karnofsky performance status of at least $60 \%$. To monitor the clinical response, the patients were required to have one or more clinically or radiologically measurable lesion. The sample size was determined using a two-stage Simon mini-max design allowing for the early termination of the study should the treatment show insufficient activity [16]. According to the Simon design, 30 patients eligible for clinical response were required for stage 1. If four or less responses were observed in these patients, the trial would have been stopped due to the treatment having insufficient activity, otherwise the trial would continue into stage 2 , until 41 patients eligible for objective response were recruited.

The treatment consisted of gemcitabine $1000 \mathrm{mg} / \mathrm{m}^{2}$ on days 8,15 and 22 of a 28-day cycle and $13-$-cis-RA $1 \mathrm{mg} / \mathrm{kg}$ orally on days $1-14$, for six cycles. All patients were monitored for signs of any World Health Organisation defined toxicity, at weekly intervals. Unacceptable toxicity of grade 3 or 4 , other than resolving nausea and vomiting or alopecia, was grounds for stopping the study medication. All patients had computed tomography of the abdomen before study entry, after three cycles and at the end of the study. A full blood count and biochemistry, a physical examination, as well as a pain assessment were carried out on days 1 and 8 of each treatment cycle. The full blood count was also carried out on days 15 and 22 . The end points included the objective response rate and median survival. The study was approved by the local ethics committee and all patients signed an informed consent.

\section{Results}

Thirty patients were registered on the study, 15 men and 15 women. The patients were recruited from three oncology centres over a period of 32 months. The median age was 65 years (range 44-79 years) and the median Karnofsky performance status was $80 \%$ (range $60-100 \%$ ). Eighteen patients had locally advanced disease, and 12 had distant metastases mainly in the liver (10) and lung (two) (Table 1). Only 20 patients were evaluable for study end points. Eight patients received one to two cycles of treatment due to rapidly progressive cancer and severe disease-related symptoms required withdrawal of the chemotherapy. One patient refused to continue treatment due to personal circumstances and one remaining patient developed an acute confusional state not related to the study medication but requiring cessation of the trial drugs. The remaining patients received an average of 4.45 treatment cycles. All
Table 1 - Patient characteristics

\begin{tabular}{ll}
\hline Male & 15 \\
Female & 15 \\
Median age & 65 (range 44-79) years \\
Karnofsky performance status & 80 (range 60-100) \\
Locally advanced cancer & 18 \\
Distant metastases & \\
$\quad$ Liver & 10 \\
$\quad$ Lung & 2 \\
Response to treatment & \\
$\quad$ Partial remission & 1 \\
$\quad$ Stable disease & 7 \\
$\quad$ Progressive disease & 12 \\
Median survival & 7.8 (range 2.6-21.6) months
\end{tabular}

patients were followed up until death. One patient achieved a partial remission, seven patients had stable disease and 12 patients developed progressive disease. The toxicity associated with the treatment was mainly haematological, with eight cases of grade 3 and four cases of grade 4 neutropenia, thrombocytopenia and anaemia (Table 2). The growth factors were not used and all the cases resolved with intravenous antibiotics, supportive use of platelets and blood transfusion. None of the serious adverse events reported was caused by the study medication. 13-cis-RA was tolerated well, with a few patients complaining of dry skin, but none of the cases was beyond grade 1 World Health Organisation toxicity. The median survival was estimated at 7.8 months (range 2.6-21 months) (Table 1).

\section{Discussion}

Pancreatic tumours have a poor prognosis, even after curative resection. Gemcitabine is established as the standard treatment, having shown superiority to 5 -fluorouracil in a randomised study [2]. New chemotherapy combinations are based around gemcitabine plus other agents in patients with advanced pancreatic tumours. The combinations of gemcitabine and capecitabine, gemcitabine and oxaliplatin and gemcitabine with erlotinib have recently produced more encouraging response rates than either of them alone.

Another approach is to induce differentiation in cancer cells in the hope of increasing the sensitivity to normal apoptotic mechanisms as well as that induced by the chemotherapeutic agents. There is considerable pre-clinical and clinical data for the anti-cancer activity of different retinoids. The most established treatment is ATRA, used for promyelocytic leukaemia, as well as for cancer prevention in leukoplakia, actinic keratosis and cervical cancer (reviewed in Ref. [17]). 13-cis-RA is currently a standard treatment for leukoplakia due to preventive activity in the development of oral cancer [18]. There are also reports of studies in thyroid and prostate cancer $[19,20]$. Alitretinoid (9-cis-RA) in combination with interferon- $\alpha$ is used in Kaposi sarcoma [21] and studies have been reported in breast 
Table 2 - Toxicity during treatment

\begin{tabular}{lccc}
\hline & \multicolumn{3}{c}{ Haematological toxicity } \\
\hline Grade & Anaemia & Neutropenia & Thrombocytopenia \\
\hline 3 & 1 & 6 & 1 \\
4 & 1 & 2 & 1 \\
Non-haematological toxicity & & Constipation & Other \\
\hline Grade & Nausea and vomiting & 2 & 1 (jaundice) \\
\hline 3 & 2 & 2
\end{tabular}

cancer, renal cell carcinoma and squamous cell carcinoma [22-25]. The development of skin cancer in patients with xeroderma pigmentosum can also be dramatically reduced with preventative treatment with 13-cis-RA [26,27]. A phase III trial of 13-cis-RA in patients with neuroblastoma after the completion of chemoradiotherapy and autologous bone marrow transplantation showed an improvement in event-free survival [28], although another preventative phase III study of 13-cis-RA in non-small cell lung cancer did not prevent second malignancies. Of interest, it indicated that such treatment could be harmful in patients who smoke and beneficial in those who never smoked [29]. This may be relevant to studies in pancreatic cancer, as a high proportion of patients who develop pancreatic cancer are smokers. 13-cis-RA was also tested in established malignancies with some positive effects. A pilot study in hormone-refractory prostate cancer reported a minimal anti-tumour effect, with most patients progressing within 3 months [20]. Encouraging results were reported in cervical cancer after a pilot study of 13-cis-RA in combination with interferon- $\alpha 2$ a after radiotherapy. Such a regimen was found to be active and well tolerated [30].

Pancreatic cancer cell lines treated with a combination of gemcitabine and 9-cis-RA undergo apoptosis [14]. Studies of high concentrations of retinoids reported late apoptosis and differentiation in pancreatic cancer cells in vitrowith a marked increase in transforming growth factor- $\beta$ (TGF- $\beta$ ) [12].

Despite ample pre-clinical data on the activity of various retinoids in pancreatic cancer, the use of retinoids in the clinical setting remains controversial. Our study showed that the co-treatment of patients with advanced pancreatic cancer with 13-cis-RA and gemcitabine is feasible and well tolerated. We did not see many adverse events associated with such a biochemotherapy regimen. However, the activity of the combination was limited. The rates of response, as well as the median survival, were equivalent to the single-agent gemcitabine treatment. The lack of activity of 13-cis-RA in pancreatic cancer in vivo is probably due to the heterogeneity of pancreatic cancer cells when compared with pancreatic cancer cell lines in stable laboratory conditions. From our study, we cannot recommend the use of 13-cis-RA with gemcitabine in the treatment of pancreatic cancer. However, we cannot exclude a benefit with other chemotherapy agents that are not normally used alone in pancreatic cancer (e.g. cisplatinum). Moreover, there are a number of analogues of ATRA that may be more effective in inducing apoptosis.

Author for correspondence: A. Michael, Oncology Department, St George's Hospital, St James' Wing, Blackshaw Road, London SW17 OQT, UK. Tel: +44-1372721759; Fax: +44-2087253972; E-mail: amichael@sgul.ac.uk

Received 15 August 2006; received in revised form 23 October 2006; accepted 8 November 2006

\section{References}

1 Toms JR. CancerStats monograph. UK: Cancer Research, 2004.

2 Burris HA 3rd, Moore MJ, Andersen J, et al. Improvements in survival and clinical benefit with gemcitabine as first-line therapy for patients with advanced pancreas cancer: a randomized trial. J Clin Oncol 1997;15:2403-2413.

3 Bollag W, Majewski S, Jablonska S. Cancer combination chemotherapy with retinoids: experimental rationale. Leukemia 1994;8:1453-1457.

4 Lippman SM, Heyman RA, Kurie JM, Benner SE, Hong WK. Retinoids and chemoprevention: clinical and basic studies. J Cell Biochem Suppl 1995;22:1-10.

5 Lotan R. Retinoids and their receptors in modulation of differentiation, development, and prevention of head and neck cancers. Anticancer Res 1996;16:2415-2419.

6 Nagy L, Thomazy VA, Heyman RA, Davies PJ. Retinoid-induced apoptosis in normal and neoplastic tissues. Cell Death Differ 1998;5:11-19.

7 Evans RM. The steroid and thyroid hormone receptor superfamily. Science 1988;240:889-895.

8 Heyman RA, Mangelsdorf DJ, Dyck JA, et al. 9-cis Retinoic acid is a high affinity ligand for the retinoid X receptor. Cell 1992;68: 397-406.

9 Pettersson F, Dalgleish AG, Bissonnette RP, Colston KW. Retinoids cause apoptosis in pancreatic cancer cells via activation of RAR-gamma and altered expression of Bcl-2/Bax. Br J Cancer 2002;87:555-561.

10 Balasubramanian S, Chandraratna RA, Eckert RL. Suppression of human pancreatic cancer cell proliferation by AGN194204, an RXR-selective retinoid. Carcinogenesis 2004;25:1377-1385.

11 El-Metwally TH, Hussein MR, Pour PM, Kuszynski CA, Adrian TE. High concentrations of retinoids induce differentiation and late 
apoptosis in pancreatic cancer cells in vitro. Cancer Biol Ther 2005;4:602-611.

12 El-Metwally TH, Hussein MR, Pour PM, Kuszynski CA, Adrian TE. Natural retinoids inhibit proliferation and induce apoptosis in pancreatic cancer cells previously reported to be retinoid resistant. Cancer Biol Ther 2005;4:475-483.

13 Balasubramanian S, Chandraratna RA, Eckert RL. A novel retinoid-related molecule inhibits pancreatic cancer cell proliferation by a retinoid receptor independent mechanism via suppression of cell cycle regulatory protein function and induction of caspase-associated apoptosis. Oncogene 2005;24: 4257-4270.

14 Pettersson F, Colston KW, Dalgleish AG. Retinoic acid enhances the cytotoxic effects of gemcitabine and cisplatin in pancreatic adenocarcinoma cells. Pancreas 2001;23:273-279.

15 Brembeck FH, Schoppmeyer K, Leupold U, et al. A phase II pilot trial of 13-cis retinoic acid and interferon-alpha in patients with advanced pancreatic carcinoma. Cancer 1998;83:2317-2323.

16 Simon R. Optimal two-stage designs for phase II clinical trials. Control Clin Trials 1989;10:1-10.

17 Altucci L, Gronemeyer H. The promise of retinoids to fight against cancer. Nat Rev Cancer 2001;1:181-193.

18 Hong WK, Endicott J, Itri LM, et al. 13-cis-Retinoic acid in the treatment of oral leukoplakia. NEngl J Med 1986;315:1501-1505.

19 Grunwald F, Pakos E, Bender H, et al. Redifferentiation therapy with retinoic acid in follicular thyroid cancer. J Nucl Med 1998; 39:1555-1558.

20 Kelly WK, Osman I, Reuter VE, et al. The development of biologic end points in patients treated with differentiation agents: an experience of retinoids in prostate cancer. Clin Cancer Res 2000;6:838-846.

21 Somos S, Farkas B. Immunomodulatory treatment with low-dose interferon-alpha and oral retinoic acid in lymphangioma-like Kaposi's sarcoma. Anticancer Res 2000;20:541-545.
22 Lawrence JA, Adamson PC, Caruso R, et al. Phase I clinical trial of alitretinoin and tamoxifen in breast cancer patients: toxicity, pharmacokinetic, and biomarker evaluations. J Clin Oncol 2001; 19:2754-2763.

23 Miller WH Jr, Reyno LM, Loewen GR, et al. A phase I-II study of 9-cis retinoic acid and interferon-alpha2b in patients with advanced renal-cell carcinoma: an NCIC Clinical Trials Group study. Ann Oncol 2000;11:1387-1389.

24 Motzer RJ, Murphy BA, Bacik J, et al. Phase III trial of interferon alfa-2a with or without 13-cis-retinoic acid for patients with advanced renal cell carcinoma. J Clin Oncol 2000;18: 2972-2980.

25 Kurie JM, Lee JS, Griffin T, et al. Phase I trial of 9-cis retinoic acid in adults with solid tumors. Clin Cancer Res 1996;2: 287-293.

26 Kraemer KH, DiGiovanna JJ, Peck GL. Chemoprevention of skin cancer in xeroderma pigmentosum. J Dermatol 1992;19: 715-718.

27 Kraemer KH, DiGiovanna JJ, Moshell AN, Tarone RE, Peck GL. Prevention of skin cancer in xeroderma pigmentosum with the use of oral isotretinoin. N Engl J Med 1988;318:1633-1637.

28 Matthay KK, Villablanca JG, Seeger RC, et al. Treatment of high-risk neuroblastoma with intensive chemotherapy, radiotherapy, autologous bone marrow transplantation, and 13-cisretinoic acid. Children's Cancer Group. N Engl J Med 1999;341: 1165-1173.

29 Lippman SM, Lee JJ, Karp DD, et al. Randomized phase III intergroup trial of isotretinoin to prevent second primary tumors in stage I non-small-cell lung cancer. J Natl Cancer Inst 2001;93:605-618.

30 Park TK, Lee JP, Kim SN, Choi SM, Kudelka AP, Kavanagh JJ. Interferon-alpha 2a, 13-cis-retinoic acid and radiotherapy for locally advanced carcinoma of the cervix: a pilot study. Eur $J$ Gynaecol Oncol 1998;19:35-38. 